PREPARED FOR THE U.S. DEPARTMENT OF ENERGY, UNDER CONTRACT DE-AC02-76-CHO-3073

PPPL-3269

UC-420

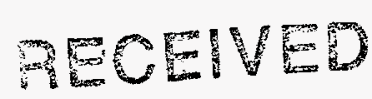

WOV 031997

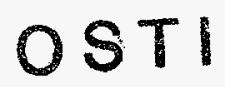

Kinetic Theory of Plasma Adiabatic Major Radius Compression in Tokamaks

by

M.V. Gorelenkova, N.N. Gorelenkov, E.A. Azizov, A.N. Romannikov, and H.W. Herrmann

October 1997

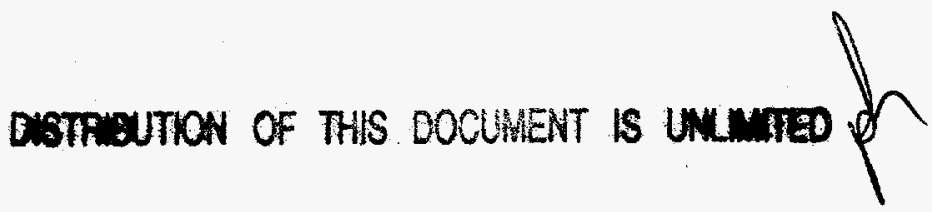

MASTER
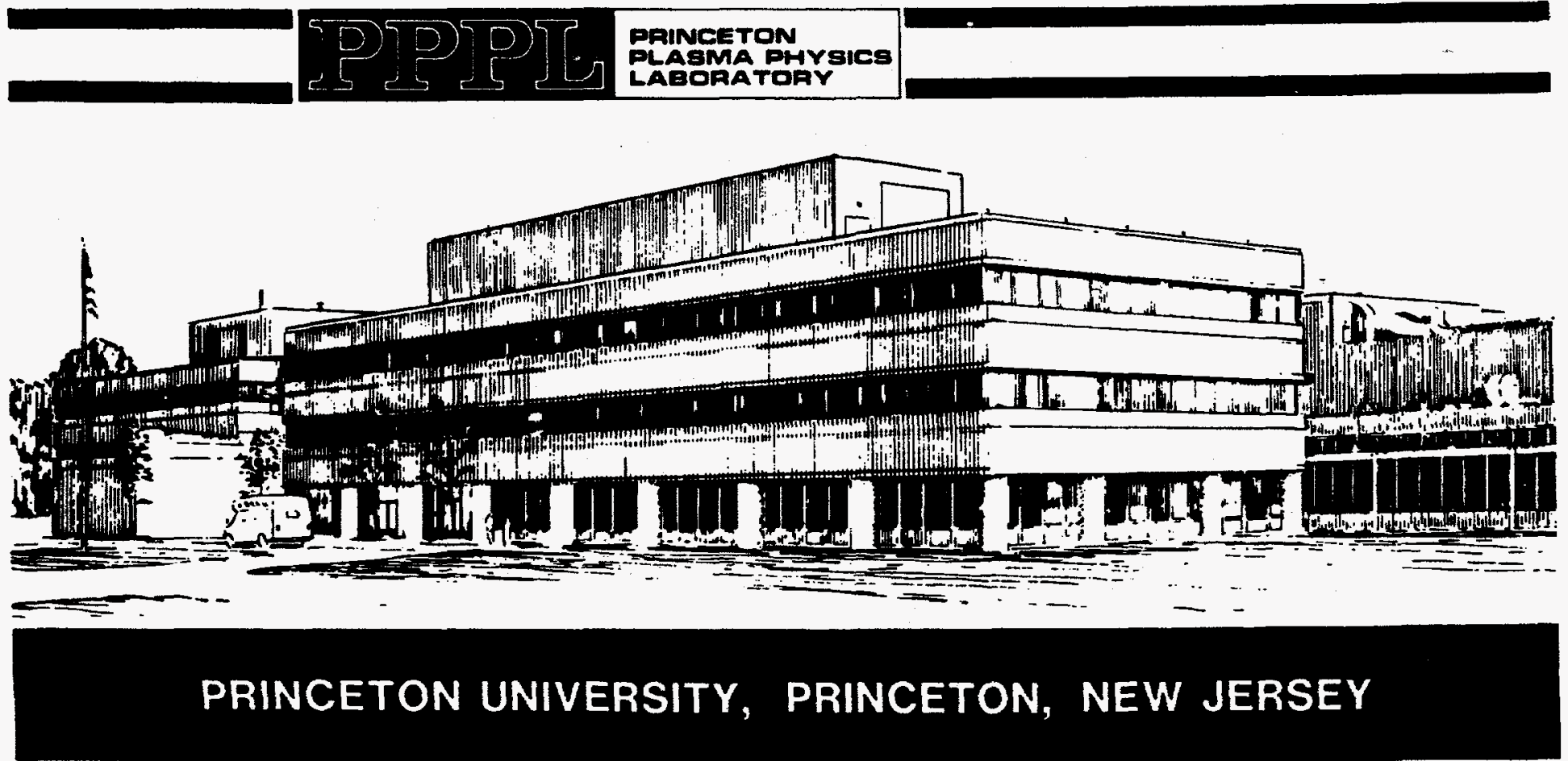


\section{PPPL Reports Disclaimer}

This report was prepared as an account of work sponsored by an agency of the United States Government. Neither the United States Government nor any agency thereof, nor any of their employees, makes any warranty, express or implied, or assumes any legal liability or responsibility for the accuracy, completeness, or usefulness of any information, apparatus, product, or process disclosed, or represents that its use would not infringe privately owned rights. Reference herein to any specific commercial produce, process, or service by trade name, trademark, manufacturer, or otherwise, does not necessarily constitute or imply its endorsement, recommendation, or favoring by the United States Government or any agency thereof. The views and opinions of authors expressed herein do not necessarily state or reflect those of the United States Government or any agency thereof.

\section{Notice}

This report has been reproduced from the best available copy.

Available in paper copy and microfiche.

Number of pages in this report: 30

U.S. Department of Energy and Department of Energy Contractors can obtain copies of this report from:

Office of Scientific and Technical Information

P.O. Box 62

Oak Ridge, TN 37831

(615) 576-8401

This report is publicly available from the:

National Technical Information Service

Department of Commerce

5285 Port Royal Road

Springfield, VA 22161

(703) $487-4650$ 


\section{DISCLAIMER}

Portions of this document may be illegible in electronic image products. Images are produced from the best available original document. 


\title{
KINETIC THEORY OF PLASMA ADIABATIC MAJOR RADIUS COMPRESSION IN TOKAMAKS.
}

\author{
M. V. Gorelenkova, N. N. Gorelenkov*, E. A. Azizov, A. N. Romannikov \\ Troitsk Institute for Innovative and Fusion Rescarch (TRINITI) \\ Troitsk, Moscow region, Russia, 142092 \\ H. W. Herrmann \\ Princcton Plasma Physics Laboratory, P.0. Box 451, Princcton, NJ 08543
}

A kinetic approach is developed to understand the individual charged particle behavior as well as plasma macro parameters (temperature, density, etc.) during the adiabatic R-compression in a tokamak. The perpendicular electric field from Ohm's law at zero resistivity $\mathbf{E}=-\mathbf{v}_{E} \times \mathrm{B} / \mathrm{c}$ is made use of to obtain the equation for particle velocity evolution in order to describe the particle motion during the R-compression. Expressions for both passing and trapped particle energy and pitch angle change are obtained for a plasma with high aspect ratio and circular magnetic surfaces. The particle behavior near the trapped passing boundary during the compression is also studied to understand the shift induced loss of alpha particles produced by D-T fusion reactions in Tokamak Fusion Test Reactor experiments. Qualitative agreement is obtained with the experiments. Solving the drift kinetic equation in the collisional case, i.e. when the collisional frequency $\nu_{\text {coll }}$ of given species exceeds the inverse compression time $\tau_{\text {compr }}^{-1}$, we obtain that the temperature and the density evolution is reduced to the MHD results $T \sim R^{-4 / 3}$ and $n \sim R^{-2}$, respectively. In the opposite case, $\nu_{\text {coll }} \ll \tau_{\text {compr }}^{-1}$, the longitudinal and perpendicular components of the temperature evolve like $T_{\|} \sim R^{-2}$ and $T_{\perp} \sim R^{-1}$. The effect of toroidicity is negligible in both cases.

*Present address: Princeton Plasma Physics Laboratory, P.0. Box 451, Princeton, NJ 08543 


\section{INTRODUCTION}

Adiabatic compression is known to be one of the methods for auxiliary plasma heating in tokamak experiments [1-3]. Two basic scenarios for the compression were proposed: $a-$ compression or minor radius compression when a toroidal magnetic field is increased and a vertical magnetic field $B_{z}$ is adjusted for equilibrium, and $R$-compression when toroidal magnetic field is fixed and the plasma column is forced to decrease its major radii by changing $B_{z}$. The compression may be considered adiabatic if it is done during the time $\tau_{\text {compr }}$ longer than the Alfvén time and shorter than the energy confinement time. When the compression is collisional, i.e $\tau_{\text {compr }} \gg \tau_{\text {coll }}=\nu^{-1}$, where $\nu$ is collisional frequency, the particle distribution function is Maxwellian during the compression and Magneto Hydro Dynamic (MHD) is valid, which may be used to derive the compression scaling laws for plasma parameters (see for example [2]). However in the opposite case, $\tau_{\text {compr }} \leq \tau_{\text {coll }}=\nu^{-1}$, the distribution function is to be found from the drift kinetic equation and the conservation laws. Even in the collisional case for bulk plasma ions some species may have a nonequilibrium distribution function, such as $\alpha$-particles, Neutral Beam Injected (NBI) particles, Ion Cyclotron Resonance Heated (ICRH) particles, etc. Note, that this circumstance may be used for more effective heating if NBI ions are mostly injected tangentially to the magnetic axis, for which, as we will see, the parallel component of its velocity is $v_{\|} \sim R_{0}^{-1}$, while the perpendicular component goes like $v_{\perp} \sim R_{0}^{-1 / 2}$.

Another motivation for developing the theory of plasma compression in tokamaks is the behavior of energetic particles near the passing-trapped boundary. Even in a weak collisionallity such particles may be scattered from confined passing to unconfined trapped and contribute to the prompt loss flux to the first wall, which may be used as a fast particle diagnostic [4]. In Ref. [4] the signal from prompt loss detectors was measured during the plasma major radius shift experiments when the shifts were done with $\tau_{\text {compr }}=80 \mathrm{ms \epsilon c}$ and the major radius shifted over $\Delta R_{0} \simeq 10 \% R_{0}$. However no clear correlations with plasma parameters and fluxes were observed. At certain conditions fluxes increase (or decrease) in comparison with the same plasma without a shift while in another plasma parameters the

fluxes change insignificantly. When the fluxes increase the unexpected "delayed" losses were also observed during the whole shifted phase of the discharge $\sim 100-200 \mathrm{msec}$, similar to 
those in DD experiments.

Based on our results, we suggest a mechanism which could be an explanation of the observations. As we will show the plasma compression may increase or decrease loss fluxes depending on plasma parameters. Confined passing particles may be forced to move closer to the separatrix boundary where they can be more easily scattered to the loss cone. Such a mechanism may effect particles even without the external shift of plasma column.

The Shafranov shift of the magnetic axis due to the finite plasma pressure may increase during the discharge. It is noticeable and may be treated as a major radius shift of the central magnetic surfaces. Thu, Shaf "delayed" losses of confined $\alpha$-particles, reported in [5].

We propose an approach to this problem introducing the perpendicular electric field from Ohm's law under the assumption of infinite conductivity, so that the plasma local velocity can be expressed through the $\mathbf{E} \times \mathbf{B}$ drift. We also will make use of the conservation of magnetic momentum $\mu$ and toroidal angular momentum $P_{\varphi}$ and calculate the change of particle velocity, which drifts in the electric field. Such an approach differs from one used earlier [6] where only the toroidal component of the electric field was taken into account.

The paper is organized as follows. In Sec.II we present the basic equations. In Sec.III the particle flux through passing-trapped boundary is obtained as a result of the Coulomb pitch angle scattering. In Sec.IV the collisional and collisionless regimes are considered to find the plasma temperature change at the compression. A summary is given in Sec.V.

\section{BASIC EQUATIONS}

\section{A. Integrals of motion}

A compression is adiabatic if the magnetic field changes slowly on time scale, $\tau_{\text {compr }}$, compared to the Alfvén time, but fast compared to the energy confinement time. Under these conditions the drift approximation for the motion of individual charged particle remains valid. Therefore, two adiabatic invariants, magnetic moment $\mu$ and the toroidal momentum $P_{\varphi}$, are conserved in an axisymmetric tokamak equilibrium:

$$
\mu=\frac{m v_{\perp}^{2}}{2 B}=\text { const }
$$




$$
P_{\varphi}=\frac{e \Psi}{2 \pi m c}-v_{\|} R=\text { const }
$$

where $e$ and $m$ are the charge and the mass of a particle, $\Psi$ is the poloidal magnetic flux, $B$ is the magnetic field strength, $v_{\|}$and $v_{\perp}$ are the parallel and perpendicular to the direction of the magnetic field components of particle velocity $v$, respectively. It is assumed that $\mathbf{v} \cdot \mathbf{B}_{\varphi} / B_{\varphi} \simeq v_{\|} \cdot$

To define the particle motion during the compression we need the evolution equation for its velocity, which is not conserved. We will derive such an equation in section II C using the electrical field $\mathbf{E}$ from the $\mathbf{E} \times \mathbf{B}$ drift and prescribing the plasma velocity vector during the compression.

\section{B. Coordinate system}

Following Ref. [7] we introduce a curvilinear $(r, \theta, \varphi)$ coordinate system which becomes the usual cylindrical $(R, Z, \varphi)$ coordinate system when major radius $R_{0} \rightarrow \infty$ (see Fig.1). Poloidal angle $\theta$ is related to the azimuthal angle $\alpha$ of the magnetic surface by the expression $\alpha=\theta-\delta(r, \theta)$; the small correction $\delta(r, \theta)$ can be chosen in a way that the force lines of the unperturbed magnetic field are straight lines in the coordinates $\theta$ and $\varphi$ (see below). The distance to the center of the magnetic surface $r=$ const from the axis of symmetry $Z$ is $R_{a x}=R_{0}+\Delta(r)$, where $R_{0}=$ const and the small quantity $\Delta$ is the Shafranov shift,

which is determined by the equilibrium condition. The relation between the cylindrical and curvilinear coordinates is

$$
R=R_{0}+\Delta(r)+r \cos (\alpha) ; Z=r \sin (\alpha) ; \alpha=\theta-\delta
$$

In our case the toroidicity is assumed to be small $\epsilon=a / R_{0} \ll 1$, i.e. the quantities $\delta$ and $\Delta / a$ are small parameters of order $\epsilon$ (where $a$ is a minor radius). According to Eq.(2) an expression for the square of the element of length $d l$ is

$$
d l^{2}=d R^{2}+d Z^{2}+R^{2} d \varphi^{2}=\sum g_{i k} d x^{i} d x^{k}
$$

where $d x^{i}=(d r, d \theta, d \varphi)$, while $g_{i k}$ is the metric tensor [7].

The magnetic field in the curvilinear coordinates is $\mathbf{B}=\left(0, B_{\theta}, B_{\varphi}\right)$, where the toroidal and poloidal components of the magnetic field are 


$$
\begin{aligned}
& B_{\varphi}=\frac{B_{0} R_{0}}{R} \\
& B_{\theta}=B_{\theta}^{0} \frac{1-(\Lambda(r)-2 \epsilon) \cos \theta}{R^{2}}
\end{aligned}
$$

where $\Lambda$ is a small asymmetry parameter that depends on the distribution of plasma pressure and current density [7]. In the absence of a known plasma pressure gradient one can use the approximate expression for $\Lambda$

$$
\Lambda(r)=\frac{3}{4} \epsilon .
$$

The relation between $\delta$ and $\Lambda$ is determined so that the equation for the lines of force

$$
\frac{d \theta}{d \varphi}=\frac{B^{2}}{B^{3}}=\frac{1}{q(r)},
$$

is independent of $\theta$ and $\varphi . B^{2}$ and $B^{3}$ are the second and third contrvariant components of the magnetic field being given by

$$
\begin{gathered}
B^{2}=\frac{B_{\theta}}{\sqrt{g_{22}}}, \\
B^{3}=\frac{B_{\varphi}}{\sqrt{g_{33}}} .
\end{gathered}
$$

It then follows from Eqs.(5) and (6) that one must choose

$$
\delta=(\Lambda-2 \epsilon) \sin \theta .
$$

Using the equations

$$
\operatorname{div} \mathbf{B}=\frac{1}{\sqrt{g}} \frac{\partial}{\partial \theta} \sqrt{g} B^{2}=0
$$

and Eq.(6) the relation between $\Delta^{\prime}=d \Delta / d r$ and $\Lambda$ is

$$
\Delta^{\prime}=\Lambda-\epsilon,
$$

where $g=\operatorname{Detg}_{i k}$.

Taking into account Eqs.(7) and (8), the nonvanishing components of the metric tensor up to $0\left(\epsilon^{2}\right)$ are 


$$
\begin{aligned}
& g_{11}=1-\frac{\epsilon}{2} \cos (\theta)+\frac{\epsilon^{2}}{16}\left(1+35 \sin \theta^{2}\right) \\
& g_{12}=\frac{3}{2} r \epsilon \sin \theta\left(1+\frac{5}{4} \epsilon \cos \theta\right) \\
& g_{22}=r^{2}\left(1+\frac{5}{4} \epsilon \cos \theta\right)^{2} \\
& g_{33}=R^{2} .
\end{aligned}
$$

\section{Particle velocity and pitch angle evolution}

The velocity of charged particles during $R$-compression changes as a result of drift in the perpendicular electric field which we introduce from the Ohm's law at zero resistivity (see Fig.(1))

$$
\mathbf{E}=-\frac{1}{c} \mathbf{v}_{E} \times \mathbf{B}
$$

where $\mathbf{v}_{E}$ is the plasma hydrodynamic velocity vector during the compression. The plasma displacement and electromagnetic field vectors in a poloidal cross section of the tokamak are presented in Fig.(1).

In cylindrical coordinates vector $\mathbf{v}_{E}$ is defined as

$$
\mathbf{v}_{E}=(\dot{R}, \dot{Z}, 0)
$$

where $\dot{A}=d A / d t$. In the curvilinear coordinates the expressions for $\mathbf{v}_{E}$ is given by

$$
\mathbf{v}_{E}=\left(v_{E r}, v_{E \theta}, 0\right)=\left(\sqrt{g_{11}} \frac{\dot{R} Z_{\theta}^{\prime}-\dot{Z} R_{\theta}^{\prime}}{R_{r}^{\prime} Z_{\theta}^{\prime}-Z_{r}^{\prime} R_{\theta}^{\prime}}, \sqrt{g_{22}} \frac{\dot{Z} R_{r}^{\prime}-\dot{R} Z_{r}^{\prime}}{R_{r}^{\prime} Z_{\theta}^{\prime}-Z_{r}^{\prime} R_{\theta}^{\prime}}, 0\right),
$$

where $F_{x}^{\prime}=\partial F / \partial x$. In order to find $\dot{R}$ we also need a time derivative or $r$, which is a consequence of the magnetic field frozen-in law,

$$
\int \mathbf{B} d \mathbf{S}=\int B_{\varphi} \sqrt{\frac{g}{g_{33}}}=\text { const } .
$$

The equation for the kinetic energy of the particle drifting in the presence of electrical field $\mathbf{E}$ is

$$
\frac{d \mathcal{E}}{d t}=e \mathbf{E} \cdot \mathbf{v}_{d r}
$$


where

$$
\mathbf{v}_{d r}=\frac{\left(v_{\|}^{2}+v_{\perp}^{2} / 2\right)}{\omega_{c}} \frac{\mathbf{B} \times \nabla B}{|B|^{2}}
$$

is particle toroidal drift velocity in the plasma low pressure approximation and $\omega_{c}$ is particle gyro-frequency. Using Eqs.(9), (10) and $p=B_{0} \mu / \mathcal{E}$ the equation for the variation of the absolute value of the particle velocity can be written in the form

$$
\frac{d v}{d t}=\frac{v}{B}\left(1-\frac{p R_{0}}{2 R}\right)\left(\frac{v_{E r}}{\sqrt{g_{11}}} B_{r}^{\prime}+\frac{v_{E \theta}}{\sqrt{g_{22}}} B_{\theta}^{\prime}-\left(\frac{B_{\theta}}{B}\right)^{2}\left(\frac{v_{E r}}{\sqrt{g_{11}}} \frac{g_{12}}{g_{22}}+\frac{v_{E \theta}}{\sqrt{g_{22}}}\right)\right) .
$$

The final expression in the curvilinear coordinates for the $d v / d t$ up to $O\left(\epsilon^{2}\right)$ accuracy is given by

$$
\frac{d v}{d t}=-v \frac{\dot{R}_{0}}{R}\left(1-\frac{p R_{0}}{2 R}\right)\left(1+\frac{\epsilon}{2} \cos \theta-\epsilon^{2}\left(\frac{5}{8}+\frac{1}{q^{2}}\right) \sin \theta^{2}\right) .
$$

Eq.(13) is a local equation. To calculate $d v / d t$ over time larger than $\tau_{b}$, which is the "bounce" time for trapped or transit time for passing particles, the Eq.(13) should be averaged over the drift particle orbit:

$$
\begin{aligned}
\left\langle\frac{d v}{d t}\right\rangle & =\frac{1}{\tau_{b}} \oint \frac{d v}{d t} \frac{d l_{\|}}{v_{\|}} \\
\tau_{b} & =\oint \frac{d l_{\|}}{v_{\|}}
\end{aligned}
$$

where $l_{\|}=\mathbf{l B} / B$. In the approximation of straight force lines in the coordinates $\theta$ and $\varphi$ (Eq.(5)), Eq.(15) reduce to

$$
\tau_{b}=\oint \frac{q R}{v_{\|}} d \theta
$$

Under the zero drift orbit radial width assumption the analytical expression of the oscillation period of trapped particles $\tau_{b}$ is

$$
\tau_{b}=\frac{8 q R_{0}}{v} \sqrt{\frac{(1+\Delta)^{2}-\epsilon^{2}}{2 p \epsilon}}[K(æ)(1+\Delta-\epsilon)+2 \epsilon E(æ)],
$$

where $K$ and $E$ are complete elliptic integrals of the first and second kind, respectively, and $æ=(1+\Delta-\epsilon)(1+\Delta+\epsilon-p) /(2 p \epsilon)$. For passing particles 


$$
\begin{aligned}
& \tau_{b}=\frac{4 q R_{0}}{v p} \sqrt{\frac{1+\Delta+\epsilon}{1+\Delta+\epsilon-p}} \\
& \times\left[K\left(\frac{1}{æ}\right)\left(2 p^{2}(1+\Delta)+\epsilon^{2}-1-\Delta\right)+E\left(\frac{1}{æ}\right)(1+\Delta-\epsilon)(1+\Delta+\epsilon-p)\right] .
\end{aligned}
$$

The expression for $d v / d t$ (Eq.(13)) for trapped particles after orbit averaging can be written in the analytical form

$$
\begin{array}{r}
\left\langle\frac{d v}{d t}\right\rangle=-v \frac{8 q \dot{R}_{0}}{\tau_{b}} \sqrt{\frac{(1+\Delta)^{2}-\epsilon^{2}}{2 p \epsilon}}\left[K(æ)\left(1-\frac{p}{2}\right)+\epsilon(K(æ)-2 E(æ))\right. \\
\left.\quad \times\left(p\left(\frac{1}{8}+\frac{1}{3 q^{2}}\right)-\left(\frac{31}{24}+\frac{1}{q^{2}}\right)+\frac{2}{3 p}\left(\frac{5}{8}+\frac{1}{q^{2}}\right)\right)\right] .
\end{array}
$$

While for passing particles

$$
\begin{aligned}
& \left\langle\frac{d v}{d t}\right\rangle=-v \frac{4 q \dot{R}_{0}}{\tau_{b}} \sqrt{\frac{1+\Delta+\epsilon}{1+\Delta+\epsilon-p}}\left[K\left(\frac{1}{æ}\right)\left(1-\frac{p}{2}\right)+\left(K\left(\frac{1}{æ}\right)-E\left(\frac{1}{æ}\right)\right)\right. \\
& \times\left(\frac{2}{3 p^{2}}\left(\frac{5}{8}+\frac{1}{q^{2}}\right)-\frac{1}{p}\left(\frac{41}{24}+\frac{5}{3 q^{2}}\right)+\left(\frac{17}{12}+\frac{4}{3 q^{2}}\right)-p\left(\frac{1}{8}+\frac{1}{3 q^{2}}\right)\right) \\
& \left.-\epsilon E\left(\frac{1}{\not 2}\right)\left(\frac{2}{3 p}\left(\frac{5}{8}+\frac{1}{q^{2}}\right)-\left(\frac{31}{24}+\frac{1}{q^{2}}\right)+p\left(\frac{1}{8}+\frac{1}{3 q^{2}}\right)\right)\right] \text {. }
\end{aligned}
$$

The pitch angle evolution equation result from the magnetic moment conservation Eq.(1):

$$
\frac{d p}{d t}=-\frac{2 p}{v}\left\langle\frac{d v}{d t}\right\rangle-p \dot{R_{0}},
$$

\section{COLLISIONAL FLUX THROUGH THE PASSING-TRAPPED BOUNDARY}

We use the equations obtained above, to calculate the change of collisional fluxes of confined fusion particles studied in major radius shift experiments [4]. Assume, that pitch angle scattering of energetic ions is a reason for a diffusion of these particles across the passing-trapped boundary. The drift kinetic equation can be presented in the following form

$$
\frac{d f}{d t}=C(f)+S
$$

where $f=f\left(v, p, P_{\varphi}, t\right)$ is the particle distribution function, $C$ represents the Coulomb collisional operator and $S=S_{0} \delta\left(v-v_{0}\right) /\left(4 \pi v_{0}^{2}\right)$ is fusion particle source at the birth velocity 
$v=v_{0}$. Assuming that the collisional effects are weak for energetic particles, an expansion in the small parameter $1 /\left(\nu_{\text {coll }} \tau_{\text {compr }}\right)$ is appropriate, $f=f_{0}+f_{1}+\ldots$, where $f_{i} / f_{i-1} \simeq$ $O\left(1 /\left(\nu_{\text {coll }} \tau_{\text {compr }}\right)\right)$. The function $f_{0}$ can be determined from the orbit average version of the first order equation

$$
\left\langle C\left(f_{0}\right)+S=0\right\rangle
$$

where $\langle\ldots\rangle$ represents orbit average operation. The model solution for the particle distribution function $f_{0}$ was found in [8] for collisional operator in the form:

$$
C\left(f_{0}\right)=\frac{1}{\tau_{s e} v^{2}} \frac{\partial}{\partial v}\left(v^{3}+v^{\star 3}\right) f_{0}+2 \frac{R}{R_{0}} \frac{v^{\star 3}}{\tau_{s e} v^{3}} \chi \frac{\partial}{\partial p} p \chi \frac{\partial}{\partial p} f_{0},
$$

where $\tau_{s e}$ is a slowing-down time, $v^{\star}=v^{\star}\left(T_{e}, n_{e}, T_{i}, n_{i}\right)$ is the critical velocity when the slowing-down rate on electrons is the same as on the plasma ion's and $\chi=v_{\|} / v=(1-$ $\left.p R_{0} / R\right)^{1 / 2}$.

Then, the particle flux across the passing-trapped boundary due to the pitch angle scattering process is obtained as follows:

$$
\Gamma=\frac{1}{v^{2}} \frac{\partial}{\partial v}\left\langle\int d^{3} v C\left(f_{0}\right)\right\rangle
$$

where $d^{3} v=d p v^{2} d v /|\chi|$. Taking into account the facts that for particles near the passingtrapped boundary layer the pitch angle scattering dominates over the energy scattering and the distribution function has a monotonically decreasing slope toward the boundary, then Eq.(18) can be written as

$$
\Gamma \simeq 2\left\langle\int \frac{d p}{|\chi|} \frac{R}{R_{0}} \frac{v^{\star 3}}{\tau_{s e} v^{3}} \chi \frac{\partial}{\partial p} p \chi \frac{f_{0}}{\Delta p}\right\rangle .
$$

Integrating Eq.(19) in $p$, we can roughly estimate the ratio of the particle flux after $R$-compression $\Gamma$ to the particle flux before compression $\Gamma_{0}$ as

$$
\frac{\Gamma}{\Gamma_{0}} \simeq \frac{\Delta p_{0}}{\Delta p} \frac{p}{p_{0}} \frac{\tau_{b 0}}{\tau_{b}(x)} \frac{g(x)}{g_{0}}
$$

where $x=R_{0}(t) / R_{0}(0), g(x)=(1+\Delta)^{2}+\epsilon^{2} / 2$ and $\Delta p$ is a solution of the equation

$$
\frac{d \Delta p}{d t}=\frac{d p}{d t}-\left.\frac{d p}{d t}\right|_{p=p s}
$$


where subscript $s$ means that the value is refered to the passing-trapped separatrix boundary and $\Delta p_{0}, \tau_{b 0}, g_{0}$ and $\epsilon_{0}$ are the parameters before the compression. Here $\Delta p_{0}$ is the characteristic width of the scattering layer near the boundary. $X$-dependence of $\epsilon$ was found from Eq.(11) and is given by

$$
\epsilon(x)=\frac{\epsilon_{0}}{\sqrt{x}}
$$

To estimate $\Delta p_{0}$ in a steady state we use the fact that the small angle deflection processes can scatter marginally confined energetic ions as they slow down. Since the velocity of the particle in the boundary layer $v$ is smaller than the birth velocity $v_{0}$, Eq.(17)is reduced to

$$
\frac{f_{0}}{\tau_{s \epsilon}} \simeq\left\langle\frac{R}{R_{0}} \chi^{2}\right\rangle \frac{v^{\star 3}}{\tau_{s e} v^{3}} \frac{f_{0} p_{0}}{\Delta p_{0}^{2}} .
$$

We also evaluate $\left\langle\frac{R}{R_{0}} \chi^{2}\right\rangle \simeq \Delta p_{0}$ and taking into accout that $p_{0} \sim 1$, therefore, $\Delta p_{0}$ can be estimated as

$$
\Delta p_{0}=\frac{v^{\star 3}}{v^{3}}
$$

\section{A. Application to major radius shift experiments in TFTR}

Our calculations show that the ratio $\Gamma / \Gamma_{0}$ is a very sensitive function of $\epsilon$. Therefore, to get a more reliable estimate of particle flux change during the $R$-compression we have to take into account the non-zero banana width. The displacement of near separatrix passing particles from the flux surface $\left(\Delta r=r-r_{\pi}\right.$, where $r_{\pi}$ is a value of $r$ on the separatrix at $\theta=\pi, v_{\|}=0$ ) has been found from the conservation laws Eq.(1) and the assumption that the displacement is small, so that we can use an expansion in the small parameter $\Delta r / r$. The solution of $r=r\left(r_{\pi}, \theta\right)$ was obtained from

$$
P_{\varphi}\left(r_{\pi}, \pi\right)=P_{\varphi}(r, \theta)
$$

keeping terms up to $(\Delta r / r)^{2}$. The safety factor profile was chosen in the form

$$
q(r)=\frac{q_{0}}{1-\left(1-\frac{q_{0}}{q_{1}}\right)\left(\frac{r}{a}\right)^{k}},
$$


where $q_{0}$ and $q_{1}$ are the edge values of $q$ at $r / a=0$ and $r / a=1$.

As an example, fig.(2) shows the dependence of the ratio $\Gamma / \Gamma_{0}$ on $\Delta p_{0}$ at different $\epsilon=$ $0.15,0.2,0.25,0.28$ for TFTR (\#86136) supershot plasma parameters [4]: $R_{0}=2.62, q_{0}=$ $1.5, q_{1}=5.1, k=1.1, x=0.9$, calculated by TRANSP code [9]. Particle energy necessary to define orbit averaging was $\mathcal{E}=2.8 \mathrm{MeV} . \epsilon$-dependence of the ratio $\Gamma / \Gamma_{0}$ is strong and is presented on Fig.(3) at different $\Delta p_{0}=0.002,0.008,0.017$. The smaller $\Delta p_{0}$ the stronger the effect. After the compression the particles flux $\Gamma$ becomes weaker compared to $\Gamma_{0}$ up to $\epsilon=\epsilon_{c r}$ when the flux $\Gamma$ exceeds $\Gamma_{0}$.

The mechanism responsible for $\epsilon$-dependence of the particle collisional flux through the passing-trapped boundary can be understood from the point of view of passing nearseparatrix particles. Fig.(4) shows the local (dashed curves) and averaged over the orbit (solid curves) velocity time derivative $d v / d t$ at $\epsilon=0.1$ and 0.3 . There is a region with $\epsilon<\epsilon_{c r}$ when the averaged value $\langle d v / d t\rangle$ is larger than the local value $d v / d t$, taken at the bounce point of the separatrix particle (poloidal angle $\theta=\pi$ ). As a consequence of the fact that $p v^{2} R_{0}=$ const, the near separatrix particles have a tendency to decrease their pitch-angle, and deviate from the boundary (see Fig.5). In the case $\epsilon>\epsilon_{c r}$ the particle pitch-angle increases, since $d v / d t$ is larger than $\langle d v / d t\rangle$, therefore passing particles approach the trapped-passing boundary as in Fig.6. The existence of the $\epsilon_{c r}$ is explained by the competition between the contributions from the toroidal and the poloidal components of the magnetic field in Eq.(13). The later increases with the minor radius and generates larger electric fields at $\theta=\pi$. The separatrix boundary in Figs.(5) and (6) is given by

$$
p_{s}(x)=1+\Delta(x)-\epsilon(x) \text {. }
$$

Major radius shifts in TFTR DT discharges were done at three values of plasma current; 1.0, 1.4 and 1.8 MA [4]. Lost partially thermalized $\alpha$-particles ( $E \simeq 2.8 \mathrm{MeV}$ ) were observed by the lost alpha detector located at the bottom of the vacuum vessel at the poloidal angle $90^{\circ}$. In the 1.0 MA discharge alpha loss normalized to the neutron flux slightly decreases after the shift $\left(\Delta R_{0}=9 \% R_{0}\right)$ in comparison with baseline discharge (without R-compression). Opposite to the 1.0 MA discharge, the alpha loss slightly increases in the 1.8 MA discharge after the shift $\left(\Delta R_{0}=5 \% R_{0}\right)$. The most pronounced effect occurs in the $1.4 \mathrm{MA}$ discharge at $10 \% \mathrm{R}$-compression. The shifted discharge displays alpha loss approximately $60 \%$ higher 
than the baseline shot.

To find the bounce point coordinates, i.e. in the experiments $\epsilon$ and $\Delta p_{0}$ for $\alpha$-particles with $E=2.8 \mathrm{MeV}$ we used Eqs. (22) and (21) at $\theta=-\pi / 2$ and the formula $v^{\star}=0.1 v_{\alpha} \sqrt{T(r)}$. The $q$ profile was approximated by the form Eq.(23) to be close to TRANSP calculated profiles, where:

$q_{0}=1.3, q_{1}=7.6, a=0.77 \mathrm{~m}, k=0.65$ for the 1.0 MA discharge;

$q_{0}=1.5, q_{1}=5.1, a=0.73 m, k=1.10$ for the $1.4 \mathrm{MA}$ discharge;

$q_{0}=1.1, q_{1}=4.9, a=0.8 \mathrm{im}, k=0.95$ for the $1.8 \mathrm{MA}$ discharge.

Therefore, we have:

$\epsilon=0.205, \Delta p_{0}=.003$ for the $1.0 \mathrm{MA}$ discharge;

$\epsilon=0.25, \Delta p_{0}=.002$ for the $1.4 \mathrm{MA}$ discharge;

$\epsilon=0.27, \Delta p_{0}=.0018$ for the $1.8 \mathrm{MA}$ discharge.

Our calculations show that for such $q$ profiles and $\Delta p_{0}$ the critical values of $\epsilon_{c r}$ are 0.21 , 0.235 and 0.265 for the 1.0 MA, 1.4 MA and 1.8 MA discharges, respectively.

Thus, it is possible to understand the experimental results. In the 1.0 MA discharge detector registers alpha particles with $\epsilon$ less than $\epsilon_{c r}$. It means that the collisional flux must decrease at the R-compression. In the case of 1.4 and $1.8 \mathrm{MA}$ discharges registered $\alpha$-particles have $\epsilon$ higher than $\epsilon_{c r}$, and the flux must increase at the compression. The ratio $\Gamma / \Gamma_{0}$ strongly dependens on the compression factor $x$ and the value of the pitch angle collisional layer width in steady state, $\Delta p_{0}$. For such experimental parameters the calculated ratios of collisional fluxes $\Gamma / \Gamma_{0}$ are $0.95(\mathcal{I}=1.0 \mathrm{MA}), 3.5(\mathcal{I}=1.4 \mathrm{MA})$ and $1.1(\mathcal{I}=1.8$ MA). Experimental data indicate that the ratio of the total particle flux in these experiments to the particle flux in similar shots without the compression are $0.8(\mathcal{I}=1.0 \mathrm{MA}), 1.6(\mathcal{I}=$ 1.4 MA), 1.2 ( $\mathcal{I}=1.8 \mathrm{MA}$ ). From the comparison of the modeling and the measurements it follows that the collisional part of the total fluxes should be: $>5 \%$ for the 1.0 MA discharge, $\simeq 17 \%$ for the $1.4 \mathrm{MA}$ discharge and $\simeq 18 \%$ for the $1.8 \mathrm{MA}$ discharge.

More detailed analysis is beyond the scope of this paper. It should include the calculation of the collisional fluxes and more accurate orbit averaging with guiding center approximation of particle orbits. 


\section{PLASMA TEMPERATURE DURING THE R-COMPRESSION}

Here we usc our approach to calculate the plasma macroparametcrs, for benchmarking the thcory with MHD and to obtain morc accuratc results for R-compression in toroidal gcometry.

\section{A. Collisional regime, $\tau_{\text {compr }} \gg \nu_{c o d l}^{-1}$}

In the case when $\tau_{\text {compr }}$ cxcceds the inverse collisional frequency $\nu_{c o l}^{-1}$ the drift kinctic cquation is reduccd to the following form:

$$
\frac{d f\left(v, p, P_{\varphi}, t\right)}{d t}=C(f)
$$

Since the collisional cffect is wcak during the orbit motion of particles, an cxpansion in small paramctcr $1 /\left(\nu_{\text {coll }} \tau_{\text {compr }}\right)$ is appropriatc, $f=f_{0}+f_{1}+\ldots, f_{i+1} / f_{i} \simeq O\left(1 /\left(\nu_{\text {coll }} \tau_{\text {compr }}\right)\right)$. Then the zcroth order in $\left.1 /\left(\nu_{\text {coll }} \tau_{\text {compr }}\right)\right)$ of Eq. $(25)$ is:

$$
C\left(f_{0}\right)=0
$$

$f_{0}$ is a Maxwcllian function as a consequence of Eq.(26). For plasma with density $n(t)$ and tempcraturc $T(t) f_{0}$ is written as

$$
f_{0}=\left(\frac{m}{2 \pi T(t)}\right)^{3 / 2} n(t) \operatorname{cxp}^{-\mathcal{E} / T(t)}
$$

The first order in $1 /\left(\nu_{\text {coll }} \tau_{\text {compr }}\right)$ cquation rcsulting from Eq.(25) is

$$
\frac{\partial f_{0}}{\partial t}+\dot{v} \frac{\partial f_{0}}{\partial v}=C\left(f_{1}\right)
$$

The cquation for the plasma density results from Eq.(28) and the particle conscrvation law

$$
\int C\left(f_{1}\right) d^{3} v d^{3} r=0
$$

Wc transform the intcgration variables in Eq.(29) from $\left(R, Z, \varphi, v_{\perp}, v_{\|}\right)$to $\left(P_{\varphi}, \Psi, \varphi, p, v\right)$. Substituting Eq.(28) into Eq.(29) and using Eq.(27) aftcr intcgrating it wc have the cxprcssion for plasma density

$$
\frac{d \ln n}{d t}=\frac{3}{2} \frac{\int 2<\dot{v}>\tau_{b} d p}{\int v \tau_{b} d p}
$$


with the integral $\int(\ldots) d p$ taken over the particle pitch angle, that is

$$
\int(\ldots) d p=2 \int_{0}^{1-\epsilon}(\ldots)_{p a s s} d p+\int_{1-\epsilon}^{1+\epsilon}(\ldots)_{t r a p} d p
$$

Eqs.(13) and (15) should be used for $\dot{v}$ and $\tau_{b}$, accordingly. Using the fact that the total energy of the system is conserved, i.e.

$$
\int C\left(f_{1}\right) \mathcal{E} d^{3} v d^{3} r=0
$$

we result in the plasma similar to Eq.(30) equation for the plasma temperature

$$
\frac{d \ln T}{d t}=\frac{2}{3} \frac{d \ln n}{d t}
$$

The dependences of the temperature growth $(\gamma(x)=d \ln T / d \ln x)$ on the compression factor $x$ at the $\epsilon=0.01,0.15,0.25,0.3$ are shown on Fig.(7). During the $R$-compression the efficiency of plasma heating decrease in edge direction ( $\epsilon$ rises), see Fig. $(8)$ where the $\epsilon$-dependence is shown at different compression factors $x=1,0.9,0.7,0.6$.

On the other hand, the expression for plasma density can be obtained from the particle conservation law

$$
N=\int n \sqrt{g} d r d \theta d \varphi
$$

and Eq.(11), which is valid in MHD. Then we have

$$
\begin{array}{r}
\frac{d \ln n}{d t}=-2+\frac{231 \epsilon^{2}+\left(64 \Delta+25 \epsilon^{2}\right) \sqrt{(1+\Delta)^{2}-\epsilon^{2}}}{160 \sqrt{(1+\Delta)^{2}-\epsilon^{2}}\left(1+\frac{13}{20} \Delta-\left(\frac{21}{5}+\Delta\right) \sqrt{(1+\Delta)^{2}-\epsilon^{2}}\right)} \\
\times \frac{1}{1+\frac{16}{21} \Delta+\frac{\epsilon^{2}}{42}(11-5 \Delta)} .
\end{array}
$$

This analytical,MHD result, and the calculated result coincide with the accuracy of used approximation $\left(\sim \epsilon^{3}\right)$.

\section{B. Collisionless regime, $\tau_{\text {compr }} \ll \nu_{\text {coll }}^{-1}$}

Plasma temperature is determinated by the distribution function $f_{0}\left(v_{0}, p_{0}, P_{\varphi 0}, t_{0}\right)$ before and by $f\left(v, p, P_{\varphi}, t\right)$ after the compression the expression for the plasma temperature is 


$$
T=2 / 3 \frac{\int \mathcal{E} f\left(v, p, P_{\varphi}, t\right) d^{3} v}{\int f\left(v, p, P_{\varphi}, t\right) d^{3} v} .
$$

In the collisionless regime, when $\tau_{\text {compr }} \ll \nu_{\text {coll }}^{-1}$ the kinetic equation is

$$
\frac{d f\left(v, p, P_{\phi}, t\right)}{d t}=0
$$

Taking into account Eq.(1), Eq.(33) gives

$$
f\left(v, p, P_{\varphi}, t\right)=f\left(v(t), p(t), P_{\varphi}\right)=f_{0}\left(v_{0}, p_{0}, P_{\varphi}, t_{0}\right) .
$$

Thus, Eq.(32) can be rewritten as

$$
T=2 / 3 \frac{\int \mathcal{E} f_{0}\left(v_{0}(v(t)), p_{0}(p(t))\right) d^{3} v}{\int f_{0}\left(v_{0}(v(t)), p_{0}(p(t))\right) d^{3} v},
$$

where the distribution function before the compression $f_{0}=f_{0}\left(v_{0}\right)$ is Maxwellian. The dependence $v_{0}=v_{0}(v(t))$ is obtained from the solution of the system of equations for $v$ and $p(\operatorname{Eqs}(13),(15)$ and $(16))$ with the boundary conditions:

$$
\begin{aligned}
& v(t=0)=v_{0}, \\
& p(t=0)=p_{0} .
\end{aligned}
$$

In the limit $\epsilon \rightarrow 0$, when only passing particles are taken into account, analytical solution of such a system is

$$
v_{0}(v, p)=v \sqrt{(1-p) x^{2}+p x} .
$$

For the plasma temperature and for longitudal $T_{\|}$and perpendicular $T_{\perp}$ components we have

$$
\begin{array}{r}
T_{\|}=\frac{T_{0}}{3 x^{2}}, T_{\perp}=\frac{2 T_{0}}{3 x}, \\
T=\frac{T_{0}}{3 x^{2}}(1+2 x) .
\end{array}
$$

The same results have been predicted in the quasi-MHD system of guiding center approximation [10]

The influence of the toroidicity $(\epsilon=0.01,0.15,0.25,0.3)$ on the plasma temperature growth obtained by the numerical calculation is presented on Figs.(9), (10) and (11) for 
longitudal, perpendicular and total temperature, accordingly. The opposite effects of the plasma heating in different directions (longitudal and perpendicular) are observed when the $\epsilon$ increases. $T_{\|}$grows more than the local $(\epsilon \rightarrow 0)$ analytical estimate gives, Fig.(9), but the efficiency of the "transverse" heating, $T_{\perp}$, decreases Fig.(10). These two effects compensate each other and $\epsilon$-dependence of total plasma temperature $T$ is weak, Fig.(11). However, efficiency of the heating may be higher if the distribution function before the compression was not Maxwellian. In fact, if we assume that the distribution is beam-like with particles mostly moving tangentially to the magnetic field, then such group of particles may be heated very effectively $T=T_{\|}=T_{0} /\left(3 x^{2}\right)$.

\section{SUMMARY AND CONCLUSIONS}

A new approach to the problem of major radius compression in a tokamak is presented based on modeling of the plasma motion during the compression by means of the $\mathbf{E} \times \mathbf{B}$ drift. The particle velocity and pitch angle evolution equations during the compression are derived.

Theory was applied to calculate the collisional fluxes through the trapped-passing boundary into the loss cone. The main results are: At $\epsilon<\epsilon_{c r}$, where $\epsilon_{c r}$ is some critical parameter, which strongly depends on plasma $q$ profile and the temperature, passing particles have a tendency to deviate from the passing-trapped boundary, while the trapped particles approach this boundary. Thus, confined counter passing particle may less effectively scatter into the loss cone after the compression. At $\epsilon>\epsilon_{c r}$ passing particles approach the passingtrapped boundary and trapped particles move away from this boundary (Fig.4). It leads to an increase in collisional losses. Presented theory may be also a candidate for the explanation of the so-called "delayed" losses observed in TFTR by S.Zweben.

In a collisional regime the adiabatic compression is less effective at higher toroidicity and the plasma temperature increases slower than at $\epsilon=0$.

In a collisionless regime plasma temperature evolution is a weak function of the toroidic-

ity. However, the efficiency of the heating during the compression may be higher in comparison with the collisional case for beam like distribution when $T=T_{\|}=T_{0} /\left(3 x^{2}\right)$. 
[1] V. E. Golant, I. P. Gladkovsky, V. A.Ipatov et al., Nucl. Fusion Suppl. 53, (1969).

[2] H. P. Furth and S. Yoshikawa, Phys. of Fluids 13, 2593 (1970).

[3] L. A. Artsimovich, Nucl. Fusion 12, 215 (1972).

[4] H. W. Herrman, Ph. D. thesis, Princeton University, 1997.

[5] S. J. Zweben, D. S. Darrow, E. D. Fredrikson, and H. E. Mynick, Nucl. Fusion 33, 705 (1993).

[6] N. N. Gorelenkov, Sov. J. Plasma Phys. 16, 241 (1990).

[7] L. E. Zakharov, V. D. Shafranov, in Reviews of Plasma Physics (Consultants Bureau, New York, 1986) Vol.11, p.153.

[8] C. S. Chang, S. J. Zweben, J. Schivell, R. V. Budny, S. Scott, Phys. Plasmas, 1, 3857 (1994).

[9] R. V. Budny, Nucl. Fusion 34, 1247 (1994)

[10] L. I. Rudakov and R. Z. Sagdeev, in: Fizika plazmy i problema UTR., (in Russian), 3, 268 (1958). 
Fig.1 Poloidal cross section $(\varphi=$ const) of the tokamak illustrating the curvilinear $(r, \theta, \varphi)$ coordinate system, electric field $\mathbf{E}=-\frac{1}{c} \mathbf{v}_{E} \times \mathbf{B}$, the plasma hydrodynamic velocity vector during the compression $\mathbf{v}_{E}$, the total magnetic field $\mathbf{B}$, toroidal $\mathbf{B}_{\varphi}$ and poloidal $\mathbf{B}_{\theta}$ magnetic field components. Plasma dimensions are defined by the major $R_{0}$ and minor $a$ radii $(r \leq a)$

Fig.2 The dependence of the ratio of the particle flux after $R$-compression $\Gamma$ to the particle flux before compression $\Gamma_{0}$ on $\Delta p_{0}$ at $\epsilon=0.15$ (solid curve), 0.2 (dashed curve), 0.25 (dashed-dot curve), 0.28 (dot curve) at the compression factor $x=0.9 . \Delta p_{0}$ is the characteristic width of the scattering layer near the boundary in steady state.

Fig.3 An $\epsilon$-dependence of the ratio of the particle flux after $R$-compression $\Gamma$ to the particle flux before compression $\Gamma_{0}$ at $\Delta p_{0}=0.002$ (solid curve), 0.008 (dashed curve) and 0.017 (dashed-dot curve) at the compression factor $x=0.9 . \Delta p_{0}$ is the characteristic width of the scattering layer near the boundary in steady state.

Fig.4 Local $d v / d t$ (dashed curve) and averaged over the drift particle orbit $\langle d v / d t\rangle$ (solid curve) values of the pre-separatrix particle velocity time derivative at $\epsilon=0.1$ and 0.3

Fig.5 The pre-separatrix particle pitch-angle behavior near the passing-trapped boundary $p_{s}$ during the R-compression as a function of compression factor $x=R_{0}(t) / R_{0}(0)$ at $\epsilon=0.1\left(\epsilon<\epsilon_{c r}\right)$

Fig.6 The same as in Fig. (5) but at $\epsilon=0.3\left(\epsilon>\epsilon_{\mathrm{cr}}\right)$

Fig.7 The power of the temperature growth $(\gamma=d \ln T / d \ln x)$ at $\mathrm{R}$-compression as a function of the compression factor $x=R_{0}(t) / R_{0}(0)$ at $\epsilon=0.01$ (solid curve), 0.15 (dashed curve), 0.25 (dashed-dot curve), 0.3 (dot curve)

Fig.8 An $\epsilon$-dependence of the power of the temperature growth $(\gamma=d \ln T / d \ln x)$ at 
different compression factors $x=1$ (solid curve), 0.9 (dashed curve), 0.7 (dashed-dot curve), 0.6 (dot curve)

Fig.9 The longitudal plasma temperature growth as a function of the compression factor $x=R_{0}(t) / R_{0}(0)$ at $\epsilon=0.01$ (solid curve), 0.15 (dashed curve), 0.25 (dashed-dot curve), 0.3 (dot curve)

Fig.10 The same as in Fig.9 but for perpendicular plasma temperature growth

Fig.11 The same as in Fig.9 but for total plasma temperature growth 


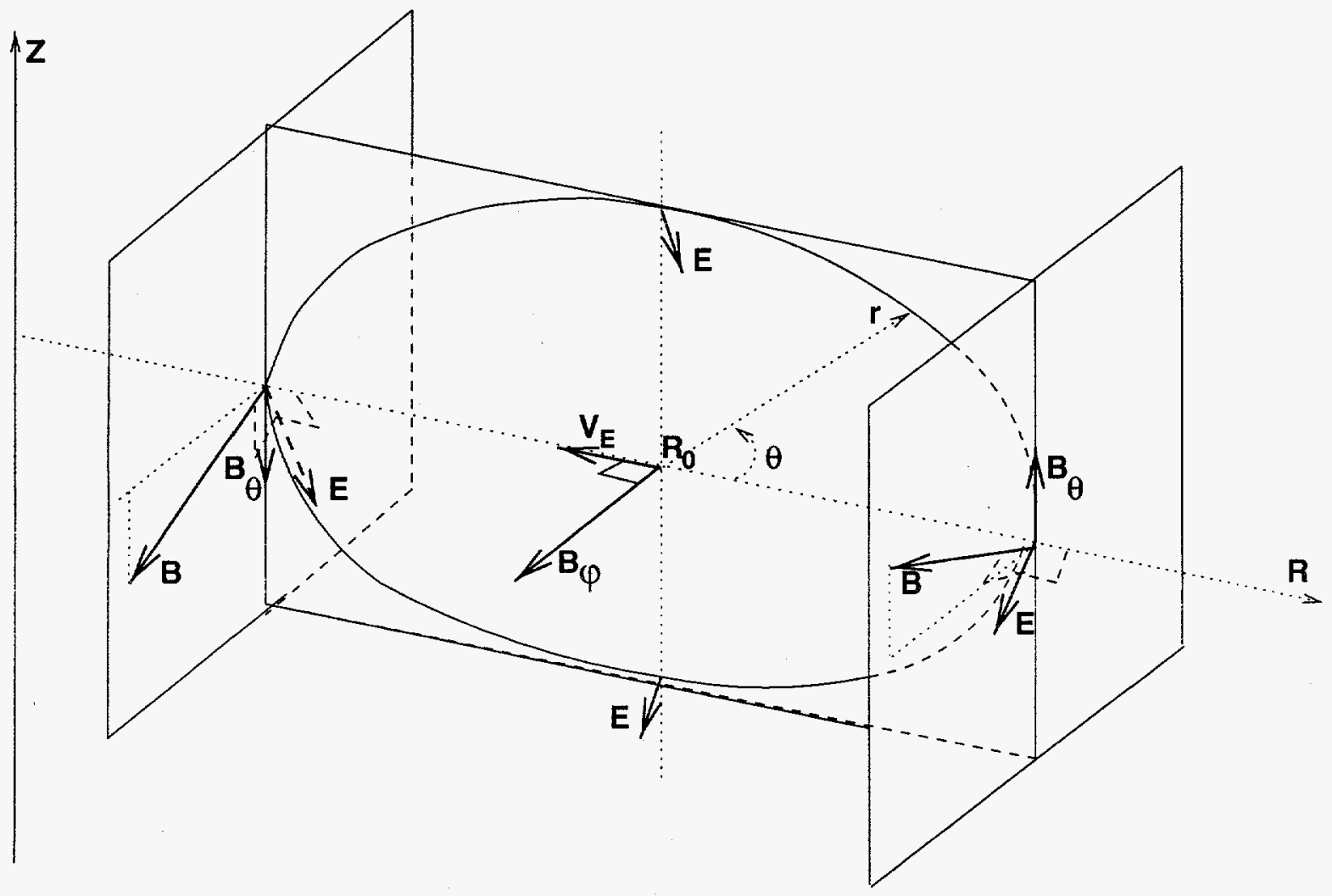

FIG. 1. 


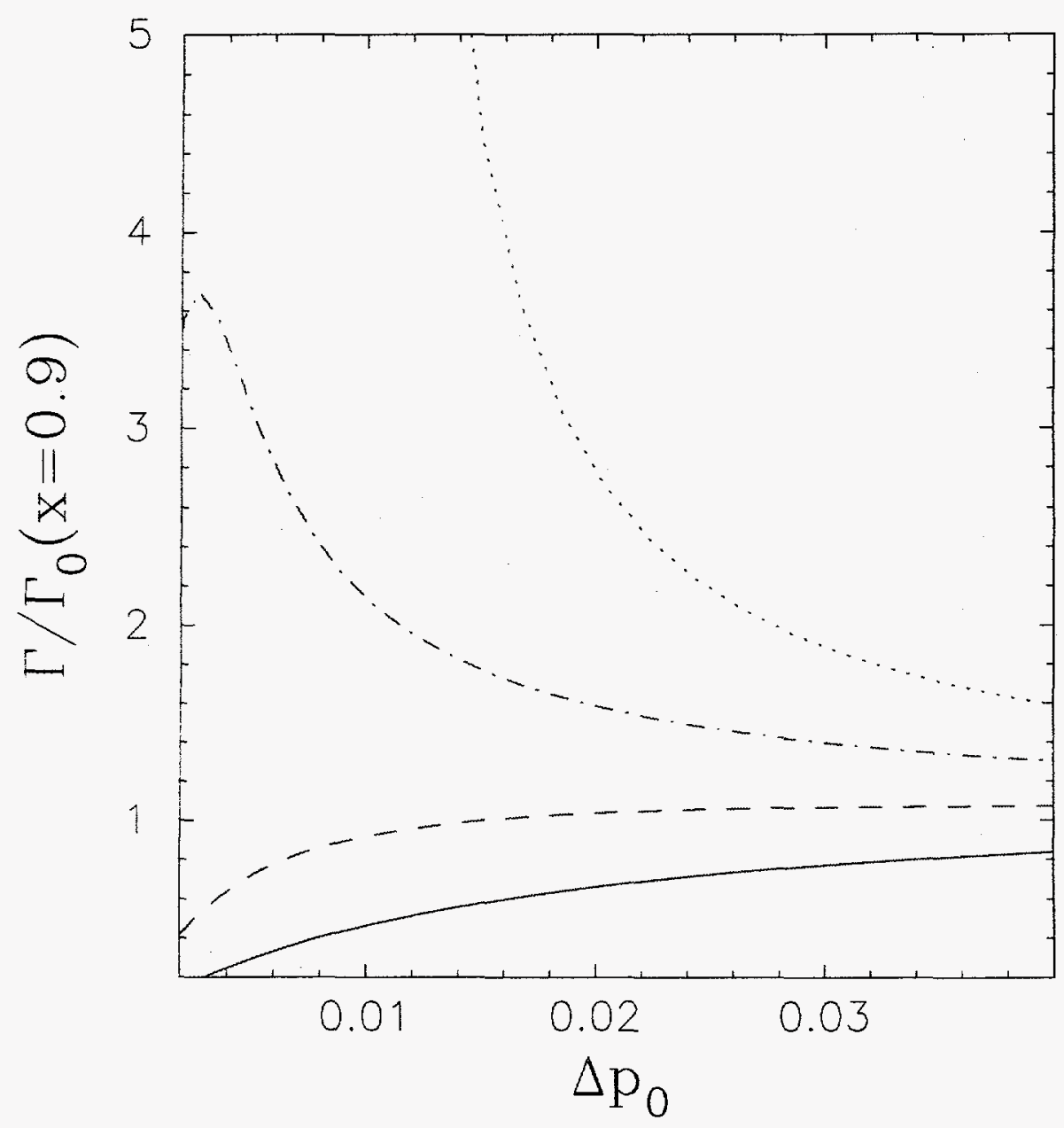

FIG. 2. 


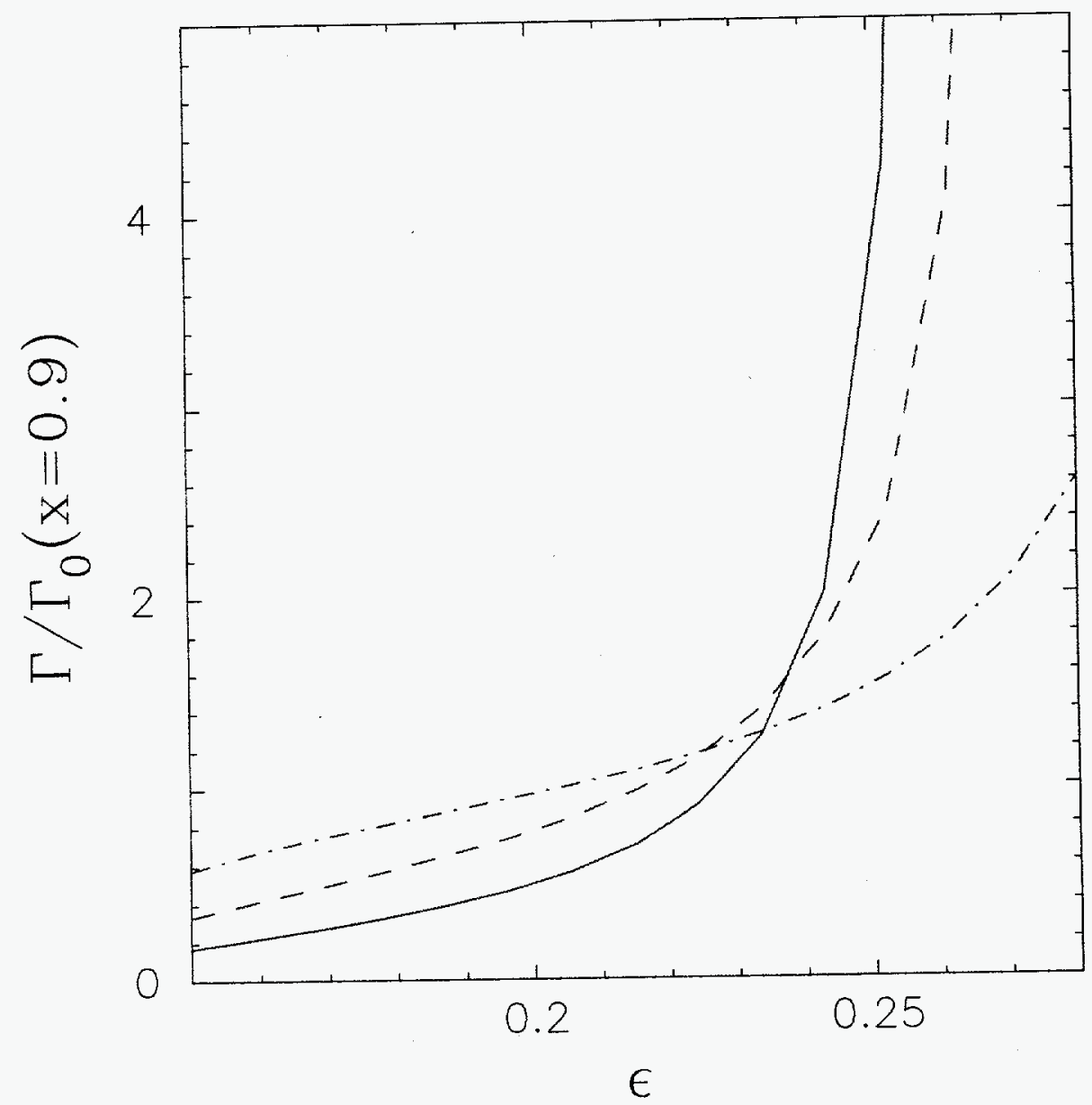

FIG. 3 . 


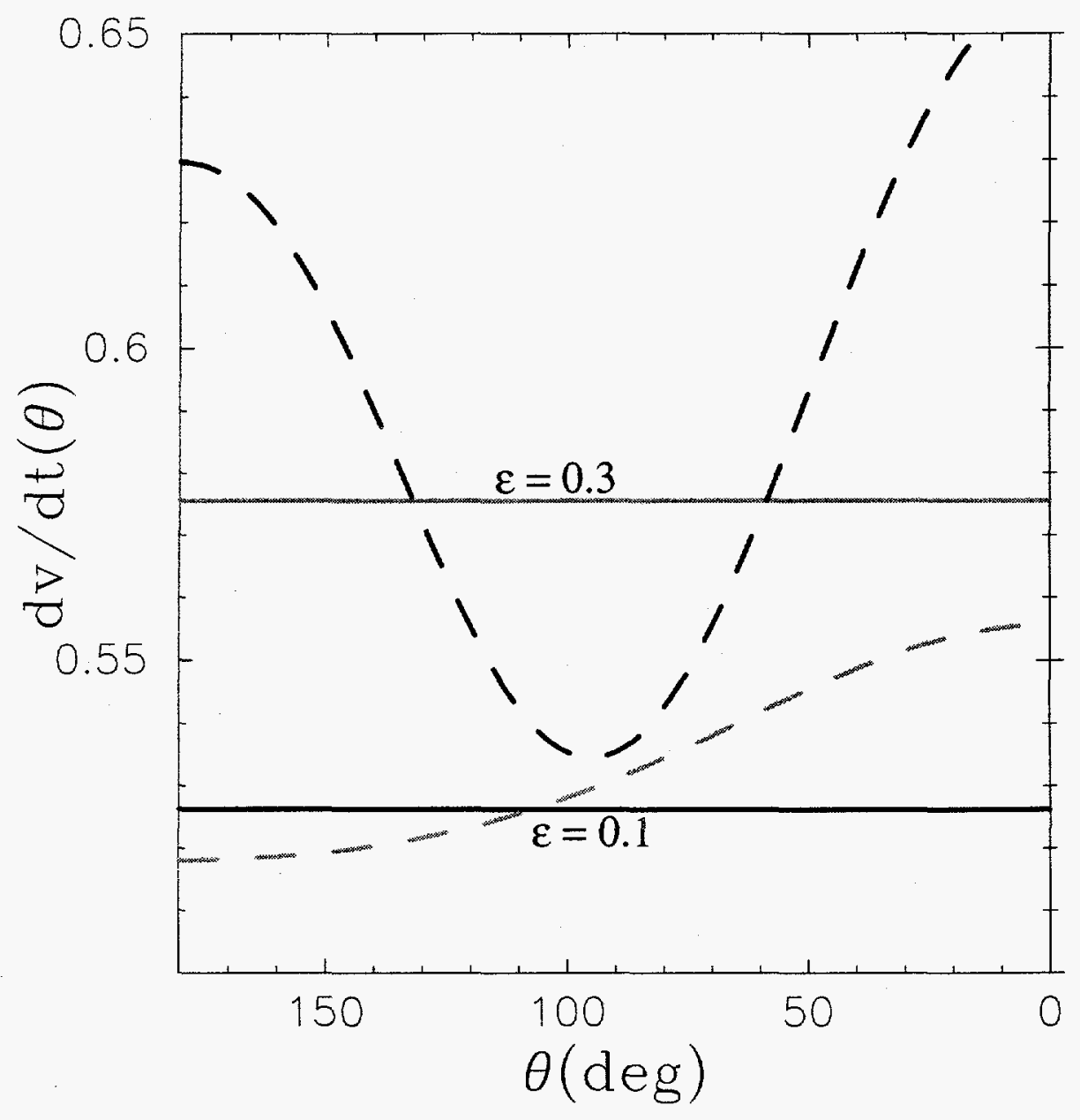

FIG. 4. 


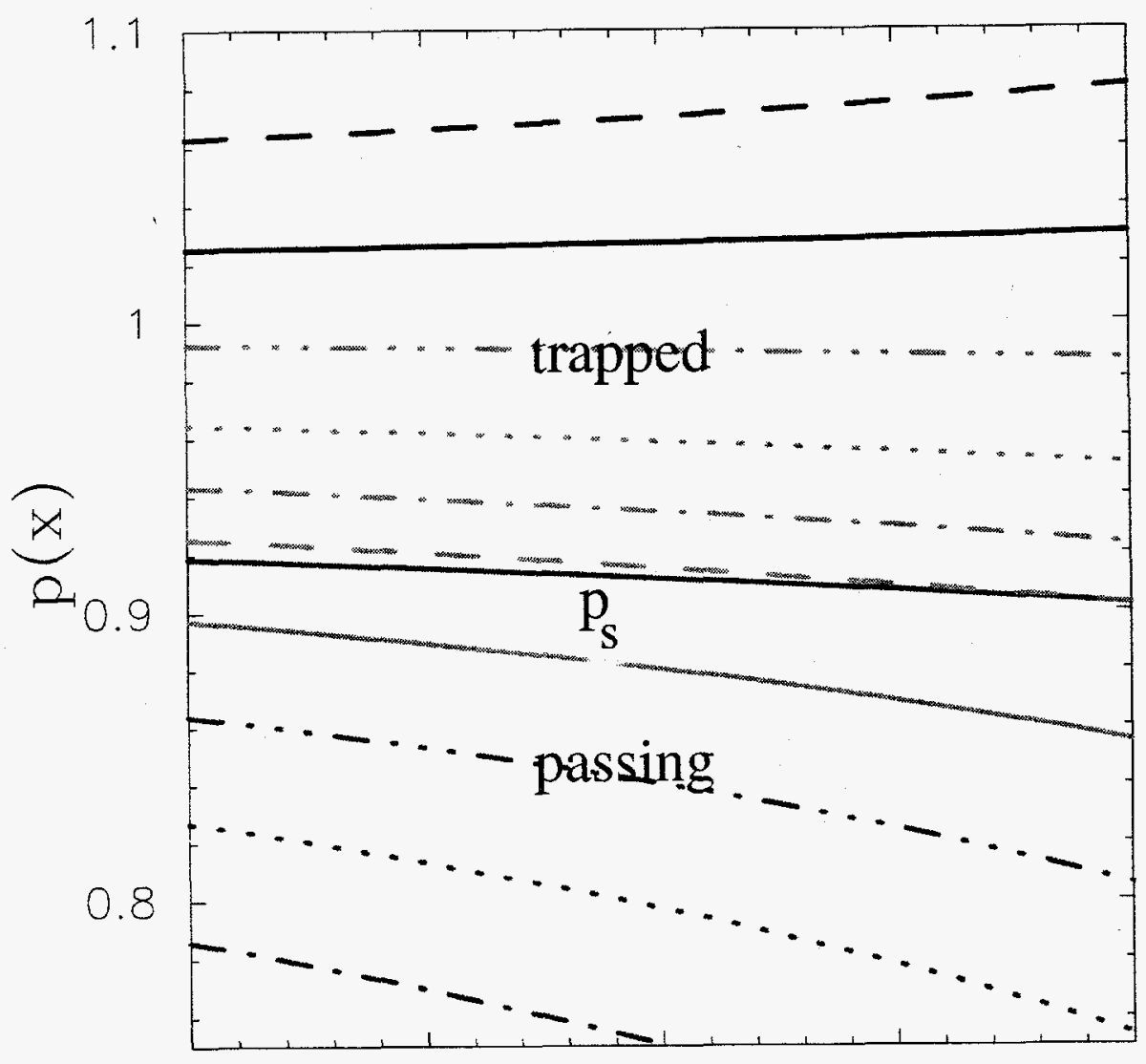

FIG. 5 . 


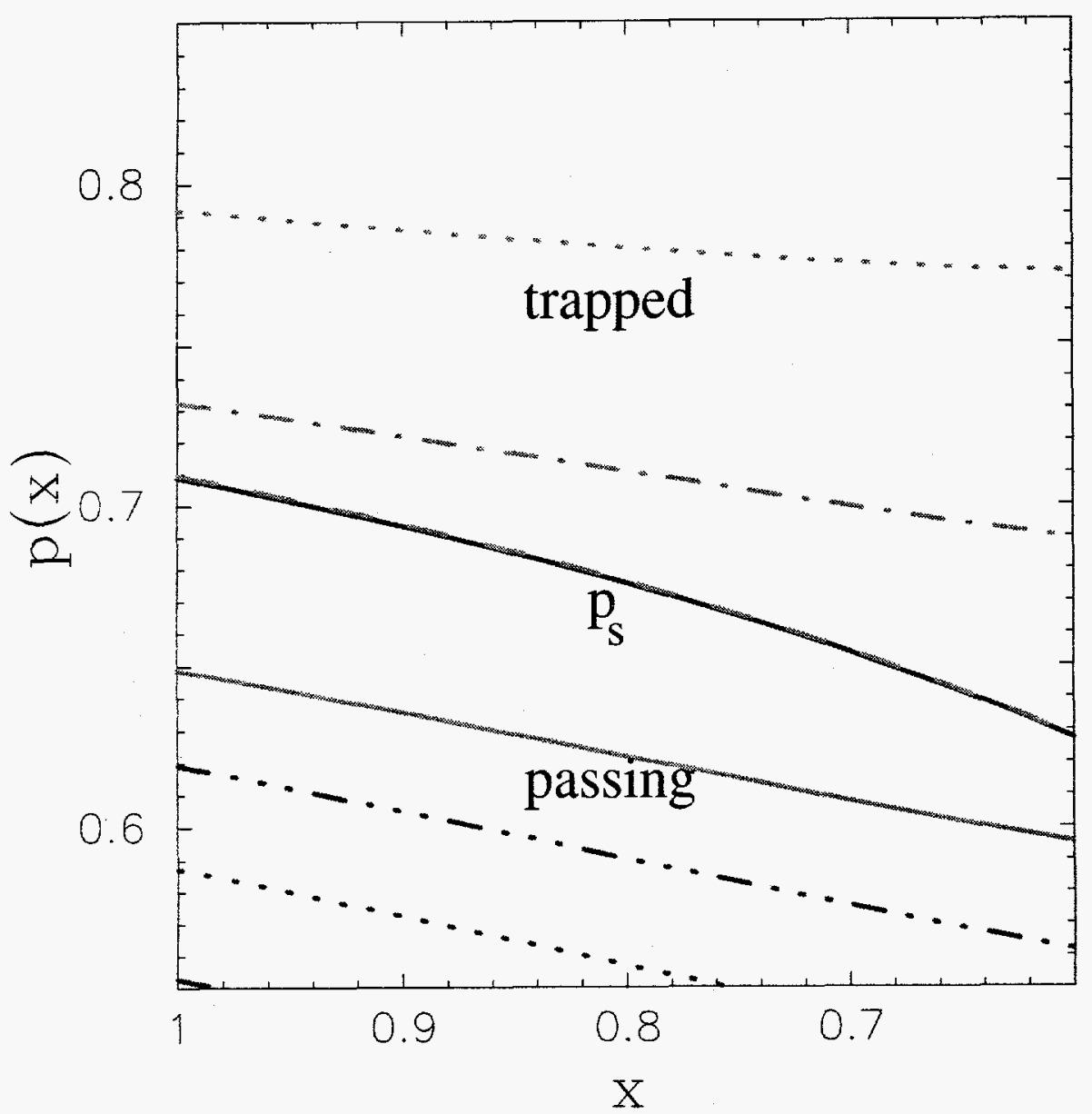

FIG. 6. 


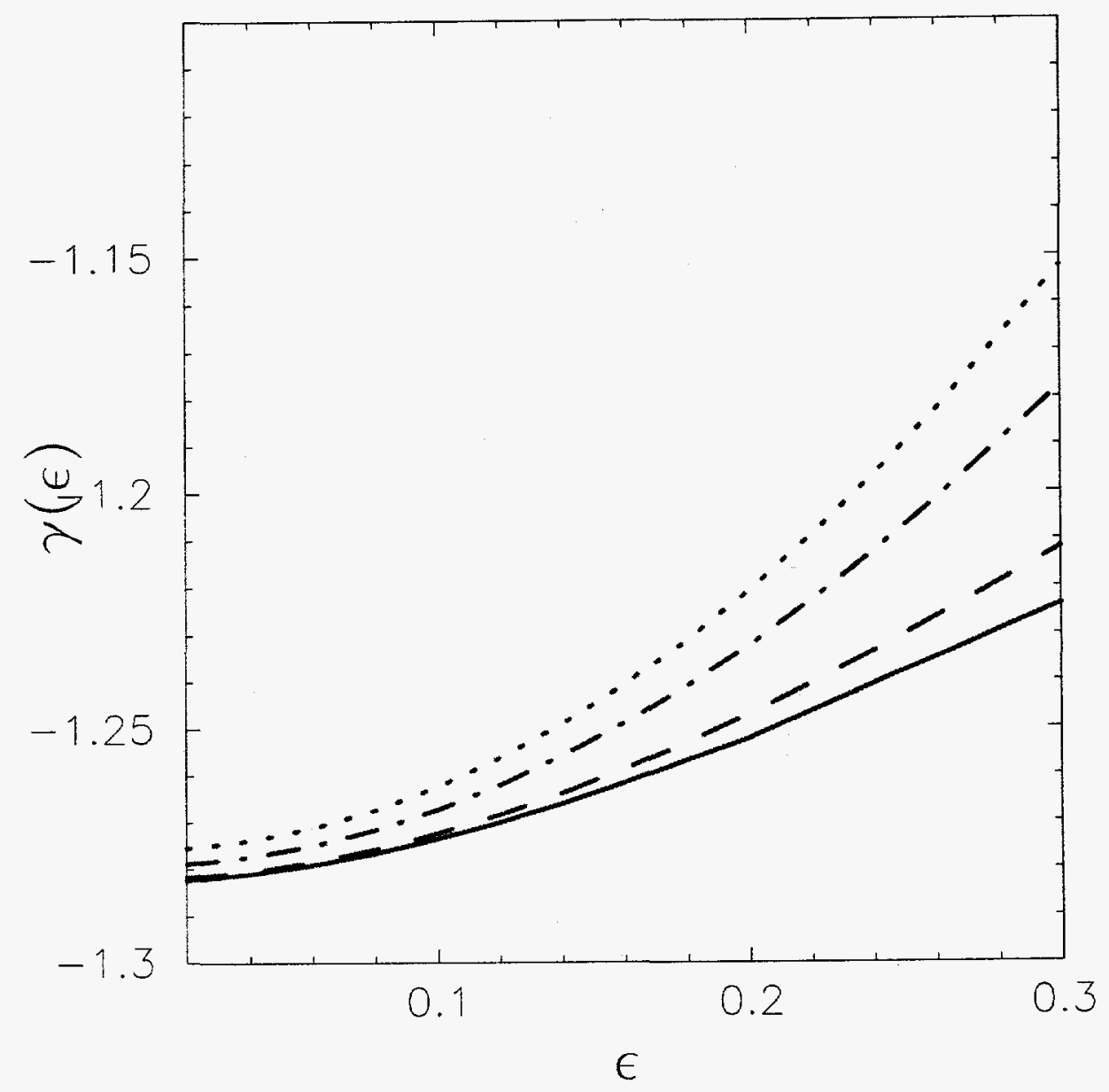

FIG. 7. 


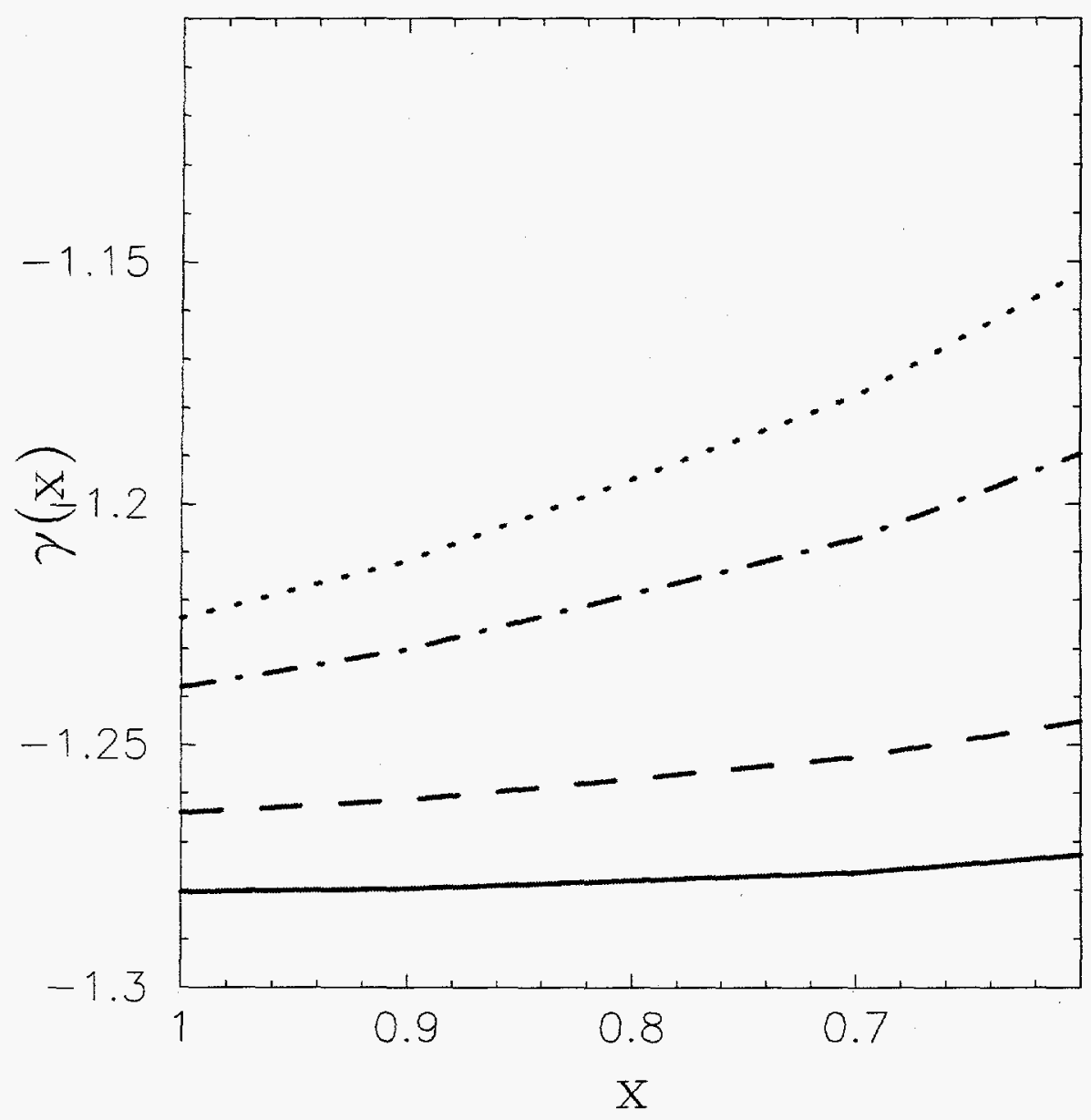

FIG. 8. 


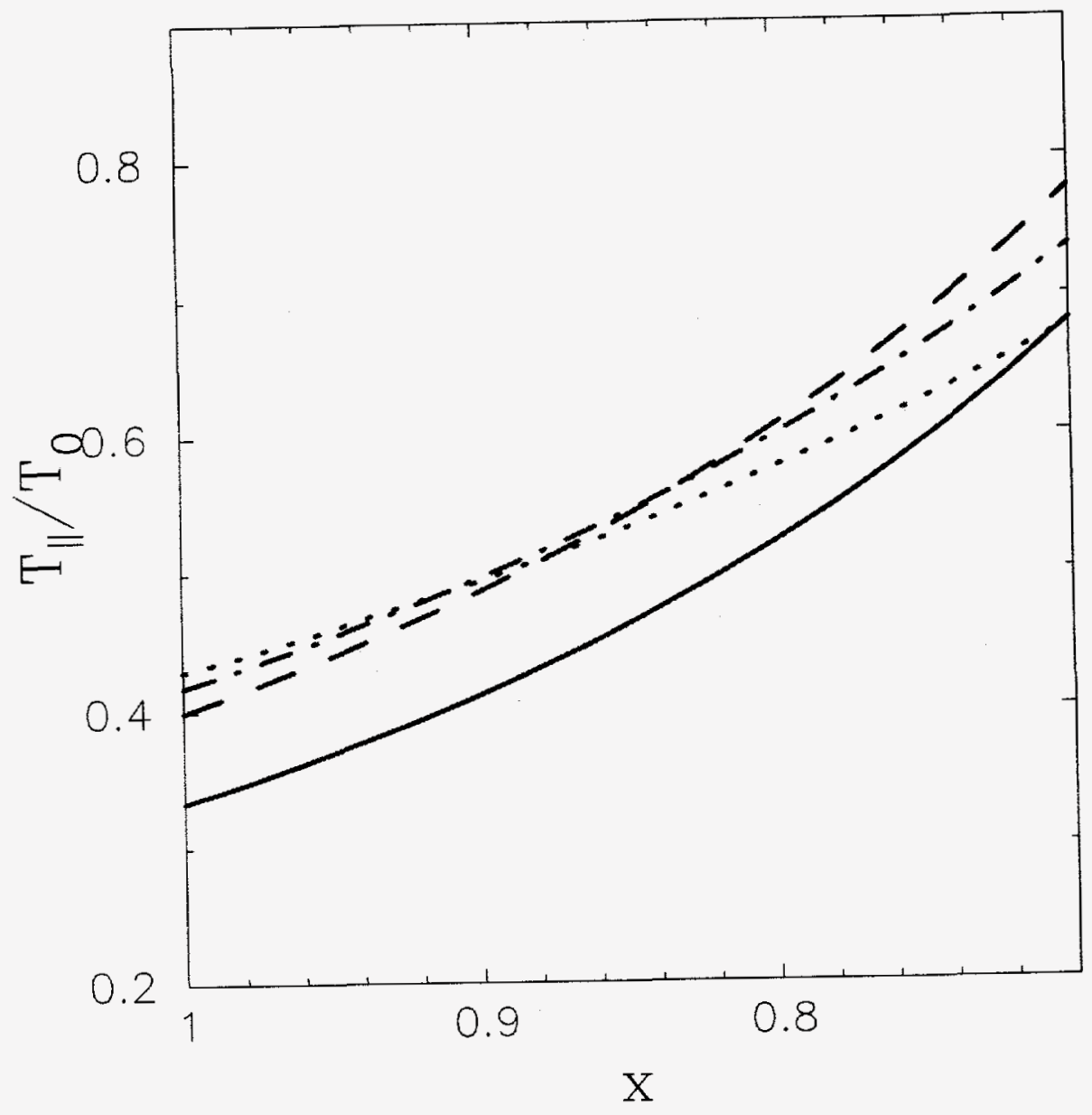

FIG. 9. 


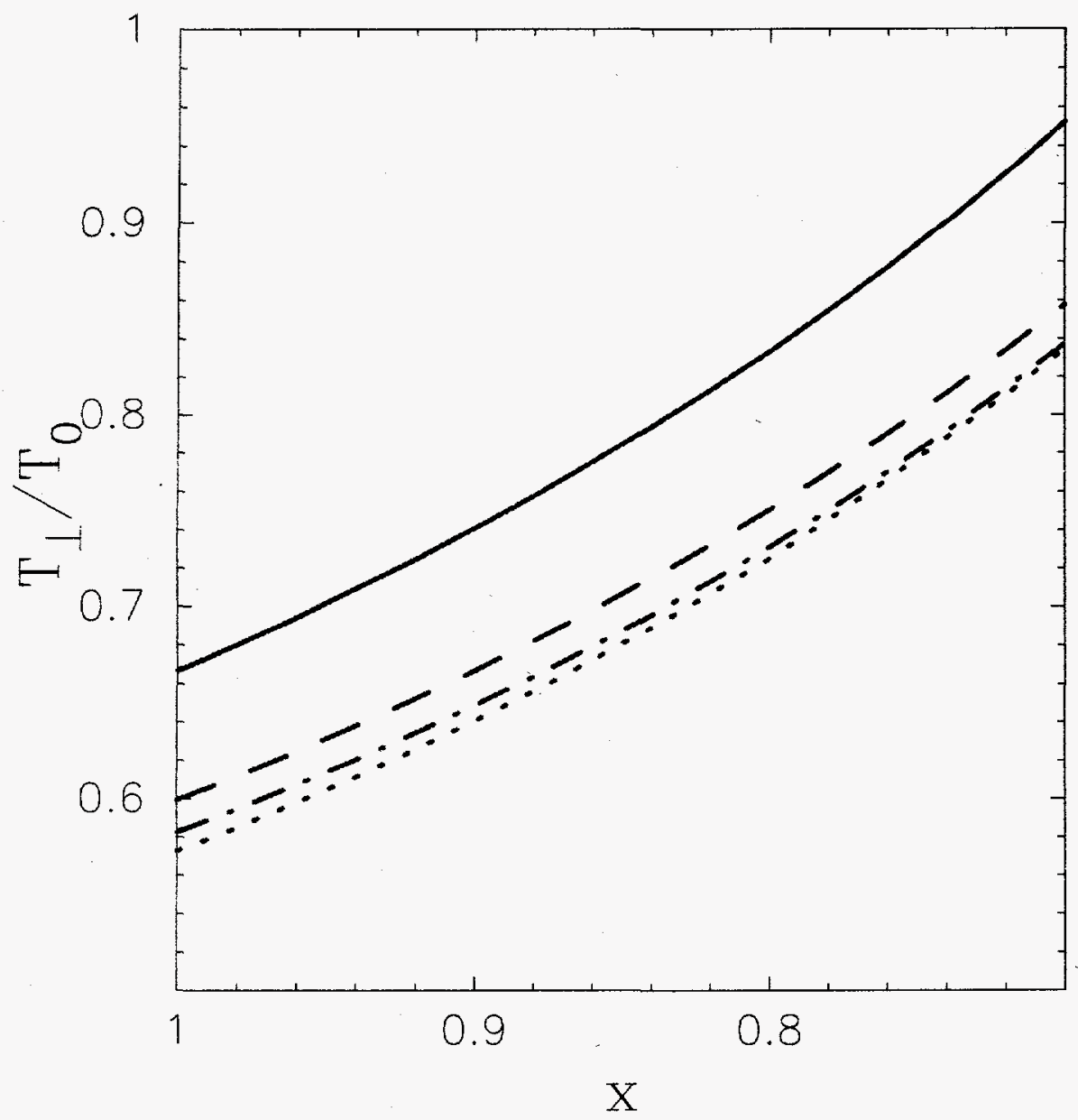

FIG. 10. 


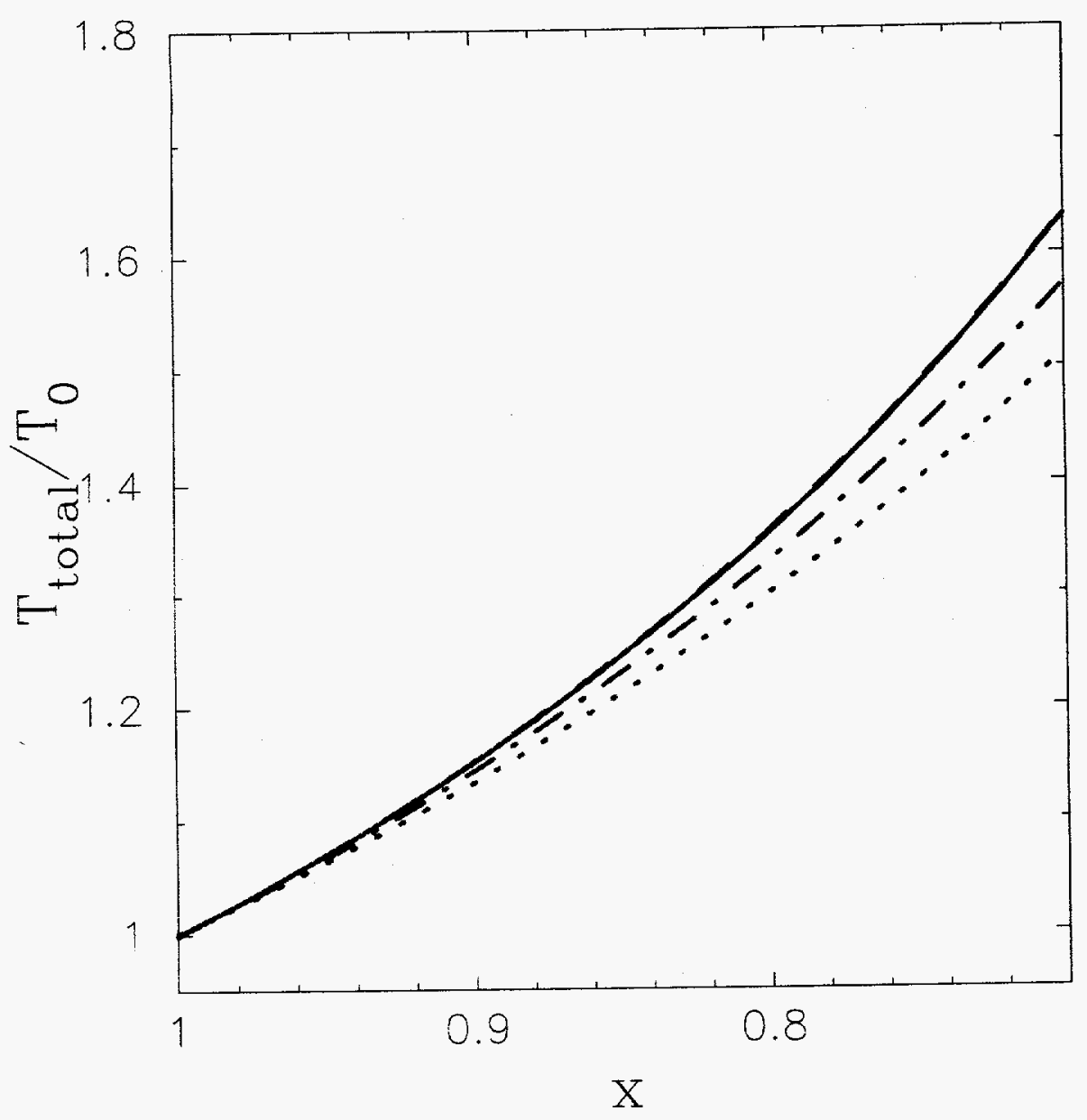

FIG. 11. 


\section{External Distribution in Addition to UC-420}

Professor Joao Canalle, Instituto de Fisica DEQ/IF - UERJ, Brazil

Mr. Gerson O. Ludwig, Instituto Nacional de Pesquisas, Brazil

Dr. P.H. Sakanaka, Instituto Fisica, Brazil

Library, R61, Rutherford Appleton Laboratory, England

The Librarian, Culham Laboratory, England

Professor M.N. Bussac, Ecole Polytechnique, France

Dr. F. Moser, Bibliothek, Institute für Plasmaforschung der Universitüt Stuttgart, Germany

Jolan Moldvai, Reports Library, MTA KFKI-ATKI, Hungary

Ms. Clelia De Palo, Associazione EURATOM-ENEA, Italy

Dr. G. Grosso, Instituto di Fisica del Plasma, Italy

Librarian, Naka Fusion Research Establishment, JAERI, Japan

Library, Plasma Physics Laboratory, Kyoto University, Japan

Dr. O. Mitarai, Kyushu Tokai University, Japan

Library, Academia Sinica, Institute of Plasma Physics, People's Republic of China

Shih-Tung Tsai, Insitute of Physics, Chinese Academy of Sciences, People's Republic of China

Dr. S. Mirnov, Triniti, Troitsk, Russian Federation, Russia

Dr. V.S. Strelkov, Kurchatov Institute, Russian Federation, Russia

Mr. Dennis Bruggink, Fusion Library, University of Wisconsin, USA

Alkesh Punjabi, Center for Fusion Research and Training, Hampton University, USA

Dr. W.M. Stacey, Fusion Research Center, Georgia Institute of Technology, USA

Mr. Paul H. Wright, Indianapolis, Indiana, USA 
The Princeton Plasma Physics Laboratory is operated by Princeton University under contract with the U.S. Department of Energy.

Information Services

Princeton Plasma Physics Laboratory

P.O. Box 451

Princeton, NJ 08543

Phone: 609-243-2750

Fax: 609-243-2751

e-mail:pppl_info@pppl.gov

Internet Address: http://www.pppl.gov 